\title{
Preface to the special edition on nano- and mesoscale friction
}

\author{
Eran Bouchbinder, Department of Chemical Physics, \\ Weizmann Institute of Science, Israel \\ Adam S. Foster, Department of Applied Physics, Aalto University, Finland \\ Oğuzhan Gürlü, Department of Physics Engineering Istanbul Technical University, \\ İstanbul, Turkey \\ Ernst Meyer, Department of Physics, University of Basel, Switzerland \\ Susan Perkin, Department of Chemistry, University of Oxford, UK \\ Andre Schirmeisen, Institute of Applied Physics, \\ Justus-Liebig-University Giessen, Germany \\ Astrid S. de Wijn, Department of Physics, Stockholm University, Sweden
}

December 16, 2015

\begin{abstract}
Recent years have seen widespread efforts to understand the mechanisms of friction and tribology in micrometric structures (mesoscale) down to the realm of atoms and molecules (nanoscale), with the ultimate goal of controlling friction, adhesion and wear by design. This research has generated an interdisciplinary scientific area, nanotribology, with great potential impact on technology and everyday life. Applications include safety, economy, life quality, energy and material saving, toward a sustainable development. Europe has a strong scientific nanotribology community encompassing physics, materials science, chemistry, earth and life sciences, and including academic and industrial research alike. This community has been expanding and collaborating for the past decades. The present special issue showcases some of the work developed in this community.

Bridging tribological mechanisms at different scales. Frictional phenomena are known to span and couple a wide range of length and time scales, and to exhibit various collective effects. Consequently, understanding friction entails a multi-scale effort. In this special issue, collective dynamics of an ensemble of macroscopic grains under shear are investigated [105208]. Possible relations to lubrication of systems at smaller scales are raised. In another work [105191], solid lubrication by graphene flakes is shown to be more effective compared to lubrication by a full graphene layer as a result of a collective mechanism that emerges from the independent behaviour of the flakes.

Tuning nanofriction. Approaches to tuning of friction on all length scales often employ lubricants and surface treatments. With current experimental techniques and numerical simulations, it has become possible to probe the nano-scale mechanisms by which these methods change the friction. In this special issue, several works investigate the effects of different structure and thickness of the tribolayer [105063,105191,105208,105396]. In [105396], Krass et al., for example, investigate how the friction depends on the number of layers of lubricant molecules in the boundary lubrication regime. Others investigate the mechanisms by which surface treatments such as patterning [105062], and coatings with specific properties [105085, 105218] can be used to change friction. There is currently also an increasing interest in changing of friction using external fields. Paronuzzi Ticco et al. [105383] investigate the effect of an oscillating external field on sliding of colloidal monolayers, with highly nontrivial results.

Confined systems under shear. Friction and lubrication between two solids is most often mediated by a thin film containing simple or complex fluid or a confined solid lubricant. The study of
\end{abstract}


these 'confined systems' under shearing conditions has been a focus of work in the nanotribology community; the molecular-level details of fluid film shear are gradually being revealed. Contained in this special issue are articles contributing to this direction of work, ranging from the molecular to the macroscopic scale. The contribution by Krass et al. [105396] describes some of the most delicate and revealing AFM nanotribology measurements yet published, detecting the shear response of hexadecane on graphite and revealing a stepwise increases in viscous and elastic response as the number of layers of alkane is sequentially reduced. Working at a very different length scale, Galmiche et al. [105063] present a new method for imaging the velocity profile of lubricant films in an elastohydrodynamic contact; a base fluid doped with a Europium dye is demonstrated to deviate from Couette flow as the pressure across the lubricant is increased.

Controlled nano movements Within the MP1303 action the research direction 'controlled nano movements' describes the predictive understanding and control of the fundamental processes underlying nanofriction and adhesion by exploiting nano-object manipulations. This strategy serves as a complementary method to study interfacial friction as a function of parameters difficult to access and modify in a standard friction force microscope. In particular, nanoparticles allow a broader choice of structurally well-defined material pairings at the interface and/or permit to vary the contact size over a wide range. Nano-objects of recurring interest are graphene flakes sliding on graphite, indeed one of the first material systems where superlubricity was shown to exist. In article [105191] the authors found in simulations superior sliding friction properties of graphene flakes stuck between two graphene layers as opposed to a full graphene layer in between them. The reason for this phenomenon is found to be related to the non-simultaneous collective translations of the flakes. In a different vein [article 105383] Paronuzzi Ticco et al. perform a theoretical analysis of Shapiro steps during sliding of monolayer under DC and AC force modulation. While this work was explicitly directed to the sliding of optical lattice trapped colloidal particle monolayers, this may also be relevant to nanoparticle manipulation under external force control.

Industrial applications. Friction is always at both ends of the spectrum for real life applications: Either it is unwanted or it is needed to be very high. For instance, the least amount of friction is required in the moving parts of a bike, yet the highest possible friction is required while making a knot to ones shoelaces before riding the same bike. The problems with friction in any industrial application are needles to be mentioned in detail. From the increase in the energy expenses, to the replacement costs of the worn-off equipment parts, friction is the central problem. Although at larger scale applications most of the problems due to unwanted friction can be solved routinely, problems due to friction at much smaller scales, such as in MEMS applications, cannot be dealt with the conventional brute force solutions of the heavy industry. This clear and present problem with friction is due to the in-existence of a complete understanding of the concept itself. Without a proper understanding, tuning friction in any application is next to impossible. Within COST action MP1303, the tasks undertaken include the reflection of the results of fundamental research to applied problems. One way of building such an information bridge is looking at the friction problem at the atomic scale and building up from there on. Investigation with proper imaging tools is a good way to start. Study of friction at sub nanometer scale with the help of atomic force microscopy gives invaluable information in the quest for cracking the puzzle on the nature of friction [105783]. The study of friction in action with further advanced imaging methodologies helps along with these efforts [105063]. Although agents that help in the reduction of friction between moving parts, possible changes in the characteristics of such chemicals in action [105208] and under confinement [105396] needs to be understood. Ultimately, rather than using intermediate materials for the reduction or the control of friction, modification of the surfaces in contact, like patterning them at the nano scale may be a pathway to tune friction [105062]. Papers in this issue reflect the multidisciplinary and multidimensional nature of this COST network. 